\title{
Occult Stage
}

National Cancer Institute

\section{Source}

National Cancer Institute. Occult Stage. NCI Thesaurus. Code C141461.

A stage term that refers to a cancer in which the primary site is unknown (not detectable by clinical or radiologic examination). The definition of occult stage depends on the particular type of cancer that it refers to; for example, for breast cancer, occult stage is defined as cancer presenting with axillary lymph node metastasis without a primary in the breast being found; for non-small cell lung carcinoma, occult stage is defined as cancer detectable by sputum cytology only and the primary tumor is undetectable radiographically or during bronchoscopy. 